\title{
Innovatives Feststoffmanagement für Wildbacheinzugsge- biete am Beispiel des Strobler Weißenbaches
}

\author{
Rolf Rindler · Patrick Holzapfel · Christoph Hauer - Gerhild Jury • Markus Moser - Andreas Fischer • \\ Clemens Gumpinger - Helmut Habersack
}

Online publiziert: 30. Januar 2019

(C) Der/die Autor(en) 2019

Zusammenfassung Der Sedimenthaushalt eines alpinen Gewässersystems bildet eine entscheidende Grundlage für dessen ökologischen und morphologischen Zustand. Gebirgsbäche stellen dabei das Bindeglied zwischen der Sedimentproduktion in den alpinen Einzugsgebieten und dem Sedimentbedarf der tieferliegenden Fließgewässer dar. Schutzwasserbauliche Maßnahmen für Wildbäche werden zur Bewirtschaftung von Feststoffen wie Geschiebe und Wildholz errichtet und je nach Bautype (z. B. Dosier- oder Filterbauwerk) ist die Wirkung auf den Sedimenthaushalt eine gänzliche Unterbrechung bis hin zum temporären Rückhalt. Bautypen mit einer gänzlichen Unterbrechung verursachen meist ein Geschiebedefizit in den flussab gelegenen Fließgewässern und mindern maßgeblich das dort vorhandene Potenzial eigendynamischer Entwicklung. Oftmals führen diese zur Eintiefung der Gewässersohle und reduzieren die Verfügbarkeit von Laichkies für die Reproduktion von Interstitiallaichern (z. B. Bachforelle). Am

DI R. Rindler $(\bowtie) \cdot$ DI P. Holzapfel . PD DI Dr. C. Hauer · G. Jury, B.Sc. . Univ.-Prof. DI Dr. H. Habersack Department für Wasser - Atmosphäre - Umwelt, Christian Doppler Labor für Sedimentforschung und -management, Institut für Wasserbau, Hydraulik und Fließgewässerforschung, Universität für Bodenkultur Wien, Muthgasse 107, 1190 Wien, Österreich rolf.rindler@boku.ac.at

DI M. Moser

Sektion Salzburg, Gebietsbauleitung Lungau, Forsttechnischer Dienst für Wildbach- und Lawinenverbauung, Johann-Löcker-Straße 3, 5580 Tamsweg, Österreich

DI A. Fischer · DI C. Gumpinger blattfisch e. U.,

Gabelsbergerstraße 7, 4600 Wels, Österreich
Strobler Weißenbach, einem typischen Kalkschuttwildbach und Zubringer zur Ischler Ache, wurden nach Hochwasserund Geschiebetransportereignissen in den 1970er-Jahren geschiebebindende Schutzbauwerke in Form von Schlitzsperren errichtet. Die Schlitze hatten das Ziel, die Feststoffe wie Geschiebe und Wildholz im Ereignisfall zurückzuhalten und bei kleineren Ereignissen wieder an den Unterlauf abzugeben. Verklausungen der Schlitze mit Wildholz/Totholz führten $\mathrm{zu}$ einem fast gänzlichen Rückhalt, wodurch sich die Verlandungsräume der Sperren immer mehr füllten. Neben der maschinellen und kostenintensiven Räumung zur Wiederherstellung der Schutzfunktion sind aufgrund der fehlenden Sedimentdurchgängigkeit auch ökologische und sedimentologische Veränderungen im Unterlauf bis in den Vorfluter die Folge. Zur Optimierung der Gesamtsituation sind Kenntnisse über die Wildbachprozesse im Jahresgang sowie bei Extremereignissen notwendig, damit einerseits die schutztechnischen Grundbedingungen erfüllt sind und andererseits auch die ökologischen und sedimentologischen Anforderungen berücksichtigt werden. Ein umfassendes Monitoringprogramm soll Erkenntnisse über die verschiedenen Prozesse am Strobler Weißenbach liefern und verschiedene biotische (Habitate, Laichplätze, Fischbestand) und abiotische Faktoren (Durchfluss, Schwebstoffkonzentration, Geschiebetransport) erfassen. Die Erkenntnisse, die aus der Studie am Strobler Weißenbach gewonnen werden, sollen in weiterer Folge als Grundlage für die Entwicklung eines allgemeinen Feststoffmanagements für Wildbacheinzugsgebiete dienen.

\section{Schlüsselwörter}

Feststoffmanagement .

Sedimenttransport · Monitoring

Tracer · Sediment Defizit

\section{Innovative concept for the management of sediments in alpine torrents}

Abstract Sediment balance and dynamics of running waters are crucial for its ecological and morphological state. Alpine rivers provide the link between sediment production in the alpine region and the need for sediment of downstream rivers. Nevertheless, protective hydraulic engineering measures like e.g. bedload retention dams may cause a total interruption in sediment continuity or at least retard sediment availability. In further consequence, downstream sediment deficit reduces the potential for morphodynamic processes, leads to deepening of the river bed, and decreases the availability of spawning gravel. However, sediment continuity is becoming increasingly important in many water management and hydraulic engineering projects. In the seventies, two slit dams were constructed at the Strobler Weißenbach, an Alpine river in the Northern Limestone Alps of Austria, because of hazard mitigation issues. Frequent clogging of the structure by woody debris leads to annual retention of huge amounts of limestone gravel Besides the downstream sediment deficit, this implies high costs in restoring the protective effect of the structure (e.g. costs for excavation, hauling or landfilling fees).

The goal of the presented study is to identify possible measures concerning the emptying of the structure considering downstream river ecology and morphology. Therefore, an extensive pre- and post-monitoring concept was implemented at the study site focusing on water level, sediment transport (PIT tag tracer, suspended sediment probes), changes in biotic data (fishing data, mapping of spawning grounds), and stream morphology (bathymetry, weighted usable area). The findings of this work should lead to the development of a novel integrative sediment 
management concept for alpine headwaters in Austria.

Keywords Sediment management Sediment transport · Monitoring . Tracer $\cdot$ Sediment deficit

\section{Einleitung}

Der Sedimenthaushalt eines alpinen Gewässersystems ist einer der wesentlichen Faktoren, welche den ökologischen und morphologischen Zustand eines Flussgebietes bestimmen. Gebirgsbäche stellen dabei das Bindeglied zwischen der Sedimentproduktion in den alpinen Einzugsgebieten und dem Sedimentbedarf der tieferliegenden Gebirgs- und Flachlandflüsse dar.

Die menschliche Wassernutzung (bspw. Wasserkraftwerke) oder auch schutzwasserbauliche Maßnahmen (bspw. Geschieberückhalt) führen stellenweise zu einer Unterbrechung dieses Sedimentflusses. Dadurch zeigt sich in vielen österreichischen Talflüssen ein Defizit an Sediment und vor allem an Geschiebe (Habersack et al. 2013). Für eine langfristig ausgeglichene und ökologisch notwendige Gewässerdynamik bzw. für eine Verbesserung des aktuellen Zustands ist eine ausreichende Sedimentverfügbarkeit aber Voraussetzung. In vielen wasserwirtschaftlichen und baulichen Planungen kommt deshalb der Sedimentdurchgängigkeit vermehrt eine hohe Bedeutung zu.

Vor allem im Wildbachbereich etablierte sich zusehends das Konzept der Geschiebebewirtschaftung, welches den vorübergehenden Geschieberückhalt während Hochwässern und die dosierte Abdrift im Nachlauf vorsieht (Suda et al. 2008). An vielen bestehenden Wildbachsperren kann eine selbstständige Entleerung des Rückhalteraums allerdings nicht gewährleistet werden. Bei bestimmten Bautypen ist eine Entleerung des Rückhalteraums nur durch maschinellen Einsatz möglich (Rudolf-Miklau and Patek 2004).

Die Erfahrungen aus der Praxis zeigen, dass die Problematik der Verlandung von Rückhalteräumen verstärkt in kalkalpinen Einzugsgebieten mit hoher Sedimentverfügbarkeit und Mobilisierung bei Starkregenereignissen auftritt. Daraus resultiert eine sukzessive Verlandung des Rückhalteraums, welcher häufig nur durch regelmäßige, technische Maßnahmen bewirtschaftet werden kann (Papež et al. 2015).
Das verlandete Volumen von Staubzw. Retentionsräumen in Österreichs Wildbacheinzugsgebieten wird aktuell auf ca. 30 Mio. Kubikmeter geschätzt und umfasst insgesamt 4864 Bauwerke, bei denen eine Geschiebebewirtschaftung notwendig ist. Pro Jahr werden zwischen 0,5 Mio und 1 Mio Kubikmeter Sedimente aus den Rückhaltebereichen entfernt, wobei die Räumkosten zwischen 12 und $18 €$ pro Kubikmeter liegen.

Aufgrund der rechtlichen Rahmenbedingungen (Abfallwirtschaftsgesetz, AWG § 3) muss das Räumgut, sobald es dem Gewässer entnommen und nicht für eine Maßnahme zur Bewirtschaftung des Gewässers verwendet wird, als Abfall verwertet bzw. behandelt werden. Obwohl nach den Grundsätzen der Europäischen Abfallrichtlinie die stoffliche Verwertung ein höherrangiges Ziel als die Beseitigung hätte, werden momentan große Massen durch Ablagerungen in Deponien beseitigt. Auch aus raumplanerischer Sicht wäre eine Remobilisierung von Sedimenten bzw. deren Verwertung zu bevorzugen (Habersack et al. 2018).

Bei einer Wiedereinbringung des Räumguts in den Bach muss allerdings gewährleistet sein, dass sich dadurch keine negativen Auswirkungen auf die Hochwassersicherheit (aufgrund von unerwünschten Anlandungen) oder den mittelfristigen ökologischen $\mathrm{Zu}$ stand des Gewässers ergeben.

Ziel der vorliegenden Arbeit ist es, die Auswirkungen von unterschiedlichen, teilweise innovativen Maßnahmen hinsichtlich der Forcierung der Sedimentdurchgängigkeit an einer der beiden Schlitzsperren am Strobler Weißenbach wissenschaftlich in einem mehrjährigen Forschungsprojekt zu untersuchen. Ein Monitoring der Wasser-, Schwebstoff- und Geschiebeführung, der Fließgewässermorphologie und der Habitatverfügbarkeit gewährleistet dabei eine objektive Erhebung der Auswirkungen und erlaubt deren Einordnung in die Bandbreite der natürlichen Dynamik (Rindler et al. 2016).

\section{Projektgebiet und Ausgangslage}

Der Strobler Weißenbach ist ein $\mathrm{Zu}$ bringer der Ischler Ache und befindet sich in den Salzburger Kalkvoralpen. Das Einzugsgebiet (EZG) umfasst eine Fläche von $45,5 \mathrm{~km}^{2}$ und grenzt im Südosten an den schroffen Höhenzug vom Gamsfeld (2027 m) bis zum Rinn- kogel (1823 m) (Abb. 1). Aus Sicht der Geologie besteht das EZG im Wesentlichen aus gebanktem Dachsteinkalk und Ramsaudolomit.

Am Strobler Weißenbach wurden zur Verringerung von Geschiebeanlandungen im Zuge von Hochwasserereignissen in den 1970er-Jahren zwei große Schlitzsperren (Kammersbachsperre und Werkstattgrabensperre) errichtet. Durch die Sperrenkonstruktion mittels Schlitz soll im Ereignisfall ein hydraulischer Rückstaueffekt erzeugt werden und somit die Geschiebe-/ Sedimenttransportkapazität stark verringern werden. Das mitgeführte Geschiebe wird im Stauraum abgelagert und bei kleineren und mittleren Hochwässern wieder in den Unterlauf abtransportiert. Verklausungen des mitgeführten Totholzes bewirkten jedoch einen ständigen Verschluss für Sediment und Geschiebe, wodurch die oben angeführte Selbstentleerung nicht mehr möglich war und die Stauräume sukzessive verlandeten (siehe Abb. 2). Laut der Auskunft von Bachanrainern sorgte über längere Zeit ein Sperrenwart für die regelmäßige Freilegung der Schlitze, was eine teilweise Selbstentleerung ermöglichte und die Funktionsfähigkeit beider Schutzbauwerke sicherstellte. Mit Wegfall dieser Tätigkeit verlandeten die Rückhaltebecken zusehends. Durch die großen Sedimentmengen stellt die Beckenentleerung eine große Herausforderung mit hohem finanziellem Aufwand und ökologischer Beeinflussung für den Unterlauf dar. Da für Bemessungsereignisse eine ausreichende Stauraumkapazität notwendig ist, muss der Schlitz entweder maschinell freigelegt oder der Stauraum geräumt werden. Nicht nur die Räumung des Materials, sondern auch die Verwertung oder Deponierung ist mit Kosten verbunden und stellt die Interessenten (Räumungsverpflichteten) oft vor eine große technische und finanzielle Herausforderung.

Im Bemessungsfall soll sämtliches potenziell schadhafte Geschiebe für den Unterlauf und Vorfluter temporär zurückgehalten werden. Daher ist darauf zu achten, dass permanent genügend Retentionsvolumen zur Verfügung steht und die Stauräume umgehend nach jedem Ereignis geräumt werden. Die Wiedereinbringung der entnommenen Sedimente in den Unterlauf ist aus mehreren Blickwinkeln sinnvoll.

Deshalb wird im Zuge des in dieser Arbeit beschriebenen Forschungspro- 


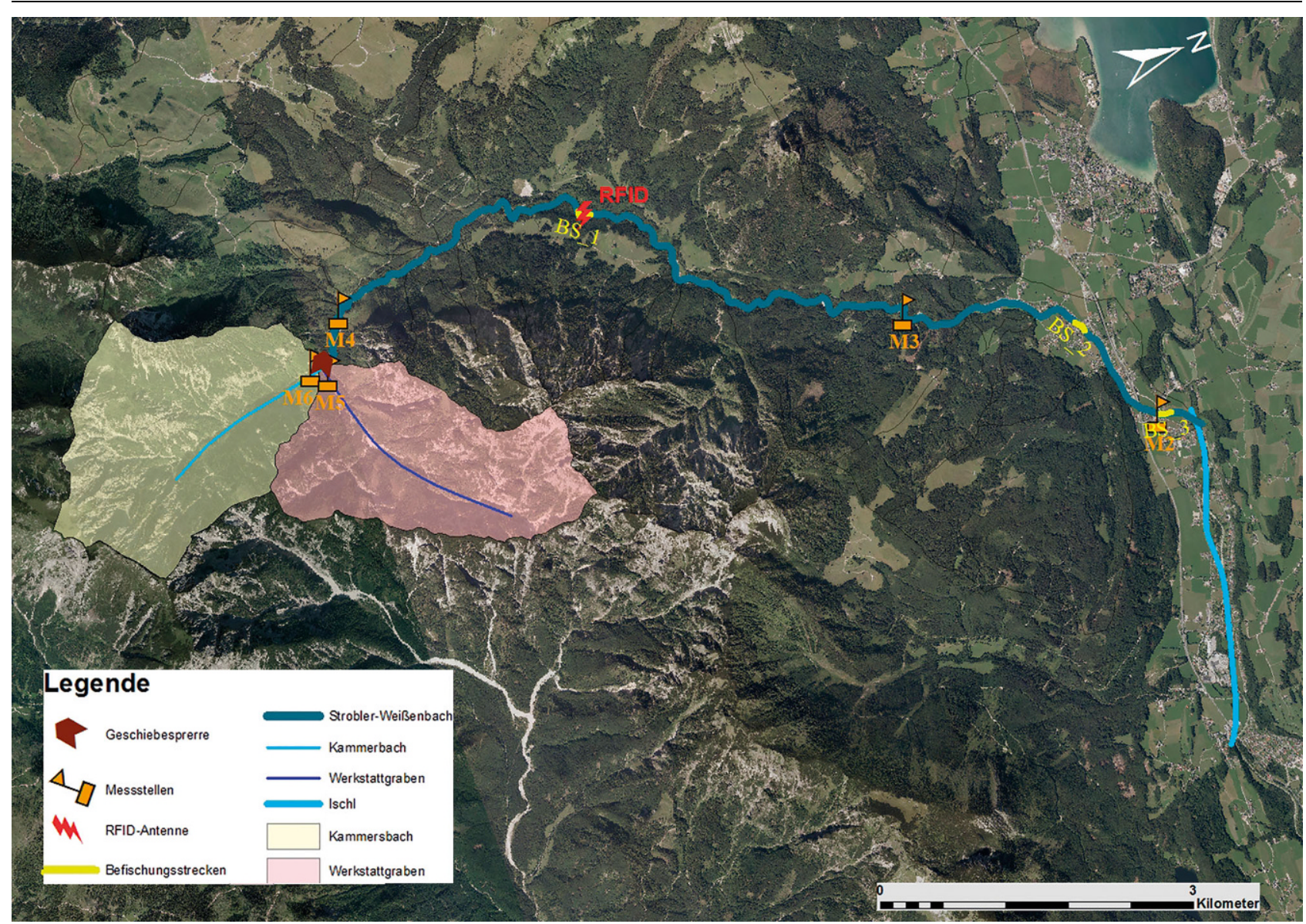

Abb. 1 Einzugsgebiet des Strobler Weißenbachs und Lage der Kammersbachsperre (BS Befischungsstrecken, $M$ Messstelle)

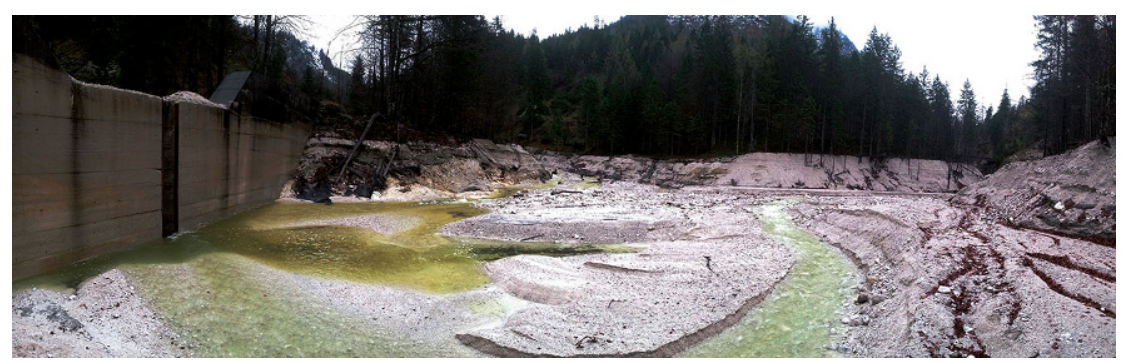

Abb. 2 Teilverlandeter Stauraum der Kammersbachsperre

jekts, ein geeignetes Geschiebemanagement entwickelt, welches einerseits versucht, die schutztechnischen Belange zu gewährleisten, aber anderseits auch die Rahmenbedingungen für ein ausgeglichenes ökologisches Gleichgewicht zu schaffen.

\section{Methodik}

Um den Ist-Zustand und die Auswirkung diverser Maßnahmen zur Stauraumbewirtschaftung der Kammersbachsperre beurteilen zu können, wurde ein umfangreiches Monitoring-Pro- befinden. In den Folgejahren wurde das Monitoring in der gleichen Qualität weitergeführt, um die Auswirkung von Eingriffen in das Sedimentregime (erhöhte Sedimentverfügbarkeit aufgrund von händischer Freilegung und Redotation von Sediment) zu validieren.

\subsubsection{Kontinuierliche Abfluss- und Schwebstofferfassung}

Um kontinuierliche Messdaten von Abfluss, Schwebstoffkonzentration und Geschiebetransport zu generieren, wurde ein umfangreiches MonitoringSystem an fünf Standorten entlang des Strobler Weißenbaches installiert (Abb. 1). Die kontinuierliche Erfassung des Wasserstands erfolgte mit keramisch-kapazitiven Drucksonden der Firma Ott, welche zusätzlich auch die Wassertemperatur erfassen. Die Ermittlung der Schwebstoffkonzentration erfolgte mit Trübungssonden (Solitax ts_line der Firma Hach Lange). In Abb. 3 ist als Beispiel die Messstation $2 \mathrm{zu}$ sehen, welche sich in etwa 


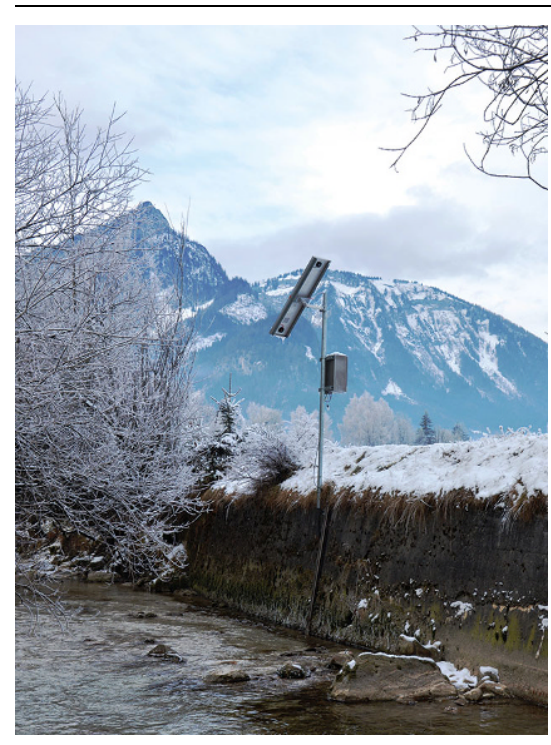

Abb. 3 Standort 2 (M2, Abb. 1) mit Wasserstandserfassung, Schwebstoffsonde und automatischer Datenübertragung

$400 \mathrm{~m}$ oberhalb der Einmündung in die Ischler Ache befindet.

\subsubsection{Bestimmung und Veränderungen des Sperrenvolumens}

Durch die Verfügbarkeit unbemannter Luftfahrzeuge (ULVZ, oft auch als Drohnen bezeichnet) besteht die Möglichkeit, mittels Fernsteuerung und GPSUnterstützung Luftbilder zu erzeugen. Am Strobler Weißenbach wird die zu untersuchende Kammersbachsperre in regelmäßigen Abständen mittels der Drohne KR 615 (siehe Abb. 4) des Instituts für Wasserwirtschaft, Hydrologie und konstruktiven Wasserbau (IWHW) in einer Flughöhe von 30 Metern über Grund beflogen. Dabei werden orthogonale Luftbilder mit einer minimalen Überlappung von $20 \%$ angefertigt. $\mathrm{Zu}-$ sätzlich wurden 30 Passpunkte (Marker) auf der Sperrenoberfläche verteilt und terrestrisch vermessen. Die fotogrammetrische Auswertung wurde mithilfe der Software „Agisoft PhotoScan Professional“ durchgeführt. Hier wurden die Bilder miteinander verbunden (aligned), idente Punkte (tie points) ermittelt, die Targets und deren Koordinaten auf den Bildern vermarkt, die Punktwolke (dense point cloud) verdichtet, eine Vermaschung (Mesh) erstellt und dadurch das Geländemodell erstellt. Um Informationen über die Änderungen der abgelagerten Kubaturen in der Kammersbachsperre zu erhalten, wurden die Geländemodelle der ver-

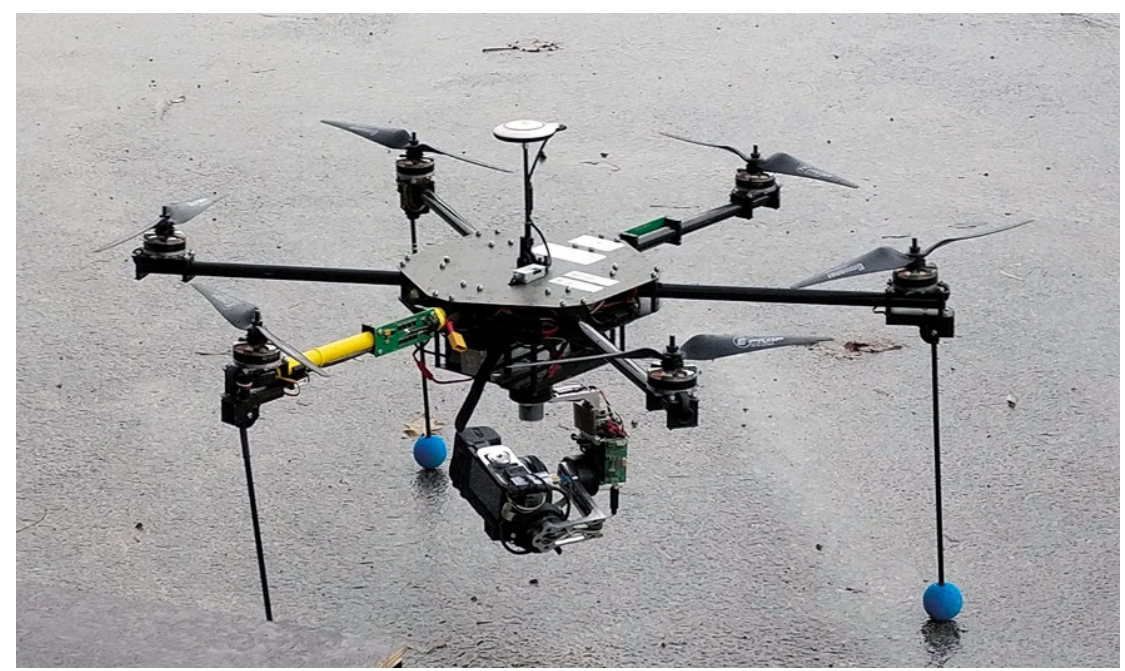

Abb. 4 Drohne KR615

schiedenen Flugtage gegenübergestellt. Dies ermöglichte eine Quantifizierung der $\mathrm{Zu}$ - bzw. Abnahme des Sedimentvolumens in der Kammersbachsperre.

\subsubsection{Lagrangesche Analyse des Geschiebetransports mithilfe von RFID-Tracern}

Die Ermittlung der Geschiebetransportweiten infolge erhöhter Wasserführung erfolgte mittels passiver Tracer (RFIDTracer der Firma Oregon). Hierfür wurden 90 natürliche Steine des Strobler Weißenbachs mit RFID-Tags besendert und am 31. August 2016 auf einer Strecke von 120 Metern zwischen Hektometer 107,6 und 106,4 wieder in den Wildbach eingebracht.

Die Dotierung der Tracer (eingeteilt in 3 Klassen „klein“ $=27-43 \mathrm{~mm}$, "mittel“ $=52-93 \mathrm{~mm}$ und „groß" $=118$ $214 \mathrm{~mm}$ ) erfolgte abwechselnd in Kolken, Furten oder Rinnen, rechtsufrig, mittig oder linksufrig in den bei Niederwasserführung benetzten Teil, um die Wahrscheinlichkeit des Transports zu erhöhen.

Die Tracersteine wurden in einem Raster mit ausreichendem Abstand zueinander eingebracht und mittels GPS verortet. Dadurch wurde der gegenseitigen Beeinflussung aufgrund sich überlagernder Signale beziehungsweise der Nichtaufzeichnung eines Signals wegen eines stärkeren Signals entgegengewirkt (Nathan Bradley and Tucker 2012). In Abb. 5 sind die Ausbringungs- orte sowie der Standort der stationären RFID-Antenne ersichtlich.

Die Tracersteine wurden bei mehreren Suchkampagnen mit mobilen Antennen detektiert und mittels GPS vermessen. Dadurch können Informationen über Transportgeschwindigkeit, Transportweiten und Ablagerungsverhalten des Geschiebes gewonnen werden. Anhand von zwei aufeinander folgenden stationären RFID-Antennen wurden und werden weiterhin alle Tracersteine detektiert, die den Untersuchungsbereich verlassen. Dies ermöglicht eine Aussage darüber, wie viele und welche Tracersteine sich noch im System befinden bzw. dieses bereits verlassen haben (Chapuis et al. 2014; Phillips and Jerolmack 2014). Durch die Installation zweier Antennen in einem Abstand von 30 Metern ist das System redundant und somit wird die Detektionswahrscheinlichkeit erhöht. Weiters ermöglicht dieses Konzept die Messung der Geschiebetransportgeschwindigkeit bei gegebenem Durchfluss. Die stationären RFID-Antennen bestehen aus einem handelsüblichen, feindrahtigen Kupferkabel, das in einem mechanisch beanspruchbaren Kabelkanal (PVC-Rohr) in Form einer rechteckigen Schleife ca. $30 \mathrm{~cm}$ tief in die Flusssohle eingebaut wurde. Als Schutz vor Erosion und Freilegung der Antenne wurden flussab zwei Reihen Steinschlichtungen als Sohlgurt in das Flussprofil eingebaut (Abb. 6). 


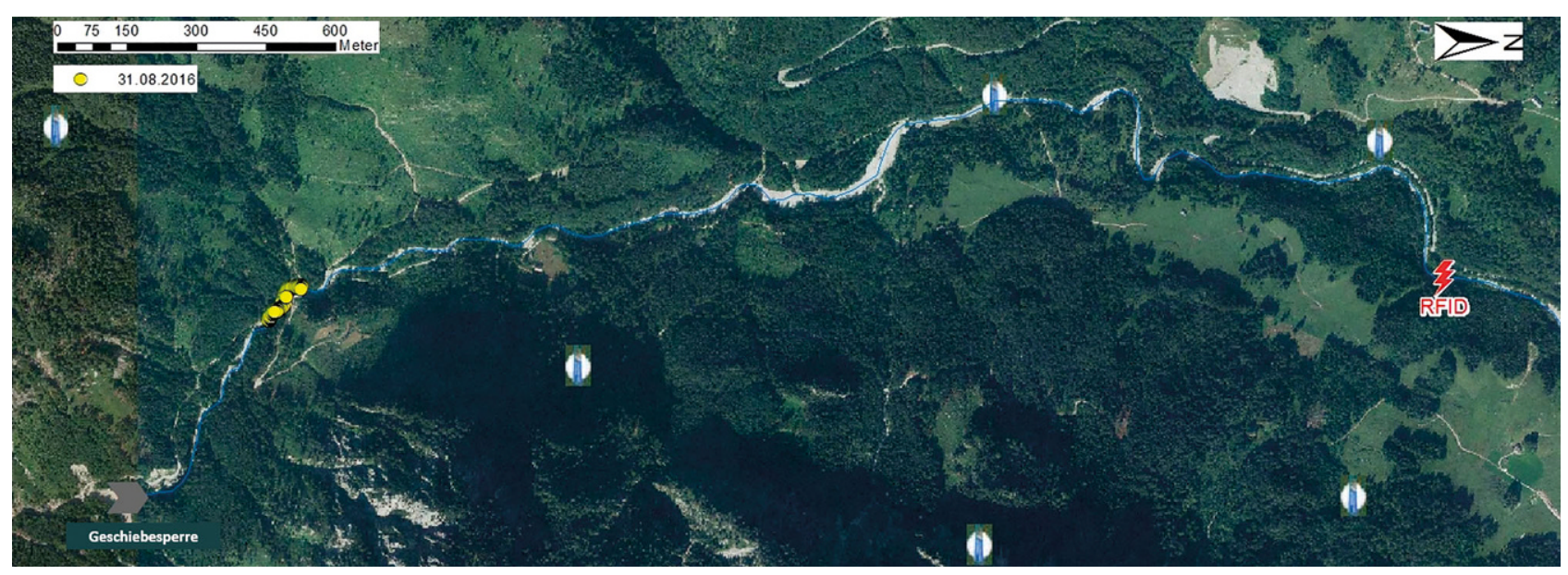

Abb. 5 Ausbringungsorte der Tracersteine

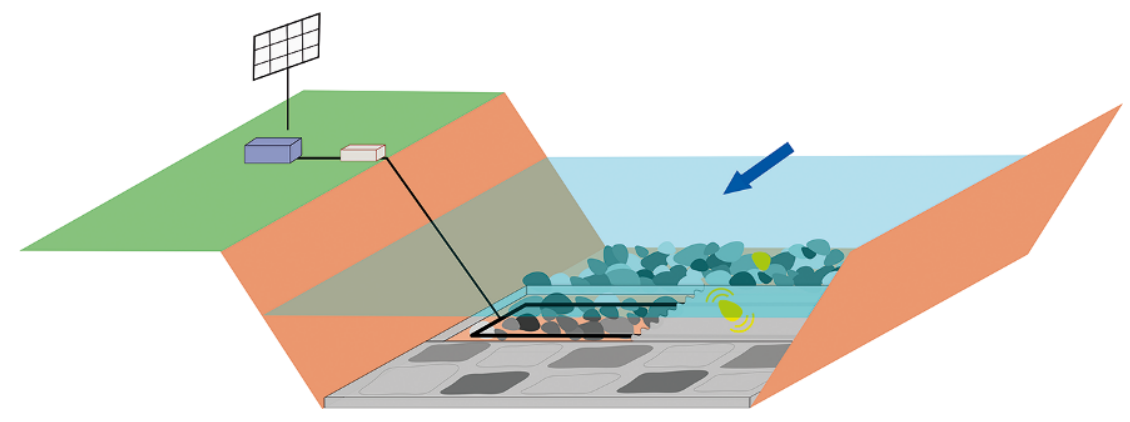

Abb. 6 Systemskizze stationärer RFID-Antennen

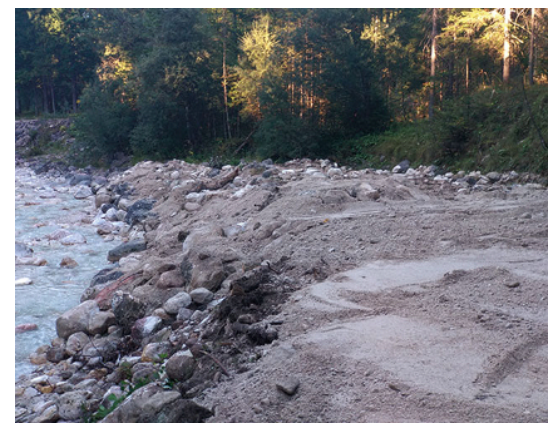

Abb. 8 Standort für die Dotierung von Sedimenten

\subsection{Sedimentzugabe}

Die Sedimentzugabe wurde in verschiedene Maßnahmen gegliedert; (i) Redynamisierung der Sedimente durch das Entfernen von Totholz, (ii) Mechanische Entnahme und Redotation der entnommenen Sedimente.

\subsubsection{Redynamisierung der Sedimente durch das Entfernen von Totholz}

In Zeiten von Niederwasserphasen wurde der Sperrenschlitz unter Berücksichtigung des optimalen Zeitpunkts aus Sicht der Ökologie ausgewählt (siehe Abschn. 3.2.3. bzw. Abb. 9) und bis $\mathrm{zu} 2 \mathrm{~m}$ freigelegt, um eine Remobilisierung der Sedimente aus der Sperre bei erhöhter Wasserführung wieder zu ermöglichen. Dies erfolgte sowohl händisch (Abb. 7), als auch mit maschineller Hilfe in Form eines Baggers.

\subsubsection{Mechanische Entnahme und Redotation der entnommenen Sedimente}

Die Redotation des entnommenen Materials erfolgte in einem Bereich mit größerem Abflussquerschnitt. Somit hat der Bach bei erhöhter Wasserführung

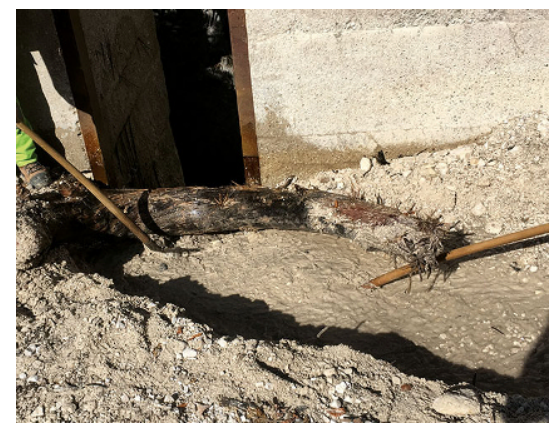

Abb. 7 Entfernen von Verklausungen (Totholzräumung) die Möglichkeit, die Sedimente selbstständig zu mobilisieren und wieder in das System einzubringen (Abb. 8).

\subsubsection{Biotik}

Im Rahmen des natürlichen Sedimentkreislaufs werden Feststoffe aus den alpinen Einzugsgebieten (Erosionsgebiete) über Wildbäche und alpine Flusslandschaften talwärts in die großen Flusslandschaften transportiert (Umlagerungs- und Ablagerungsgebiete). Der Großteil des Feststofftransports erfolgt dabei in Form von Schwebstoffen. Schwebstoffe sind Feststoffteilchen $(<0,7 \mathrm{~mm})$, welche durch die im fließenden Wasser auftretenden Turbulenzen in Schwebe gehalten werden. Sie sind natürlicher Bestandteil des Ökosystems Fließgewässer. Trotzdem können erhöhte Schwebstoffkonzentrationen unterschiedliche negative Auswirkungen auf die im Gewässer lebenden Organismen zur Folge haben. Mögliche Auswirkungen von erhöhten Schwebstoffkonzentrationen auf Salmoniden wurden von Bash et al. (2001) in folgende drei Kategorien unterteilt:

- (i) physiologische Auswirkungen (z.B. Kiementrauma, Veränderun- 


\begin{tabular}{|c|c|c|c|c|c|c|c|c|c|c|c|c|}
\hline Jun. & Jul. & Aug. & Sept. & Okt. & Nov. & Dez. & Jän. & Feb. & Mär. & Apr. & Mai & Jun. \\
\hline & \multirow{2}{*}{\multicolumn{2}{|c|}{ Juvenilstadium }} & & & aichze & & & & chlupf & & rgenz & \\
\hline & & & & \multicolumn{6}{|c|}{ Eier im Interstitial } & \multicolumn{3}{|c|}{ Larvenstadium } \\
\hline
\end{tabular}

Abb. 9 Zeittafel hinsichtlich der sensiblen Lebensphasen im Lebenszyklus der Bachforelle (sensible Phasen sind in orange Farben dargestellt)
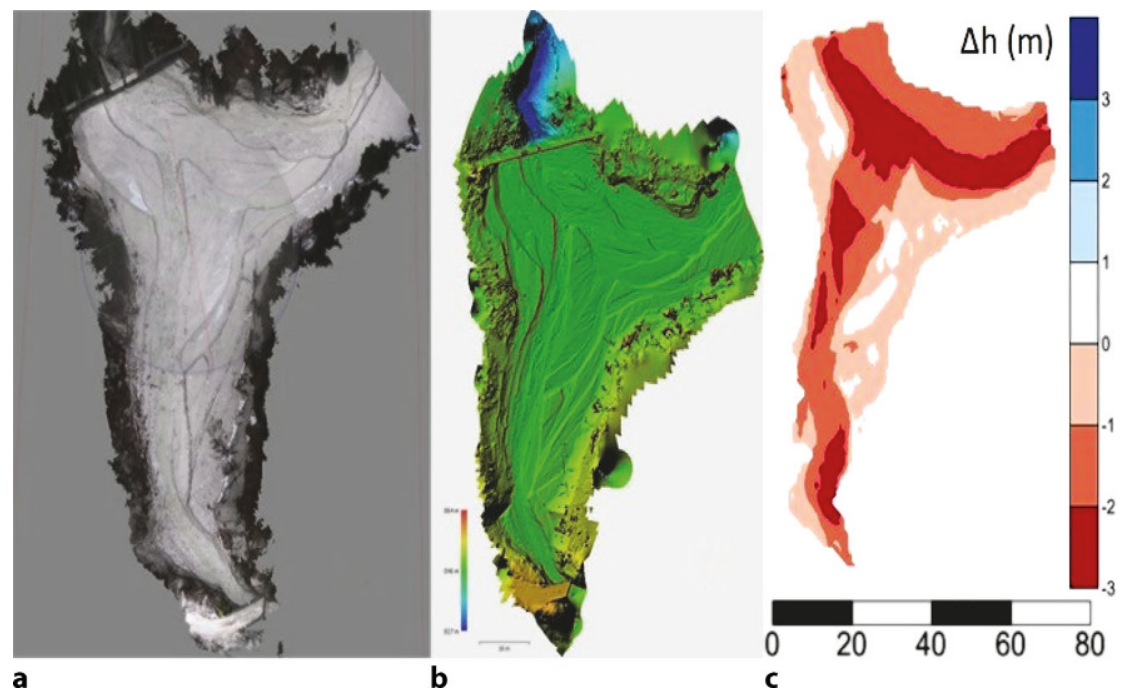

Abb. 10 a Orthofoto Kammersbachsperre, b Geländemodell 29.09.2017, c Differenzenplot 29.09.2017-16.11.2018

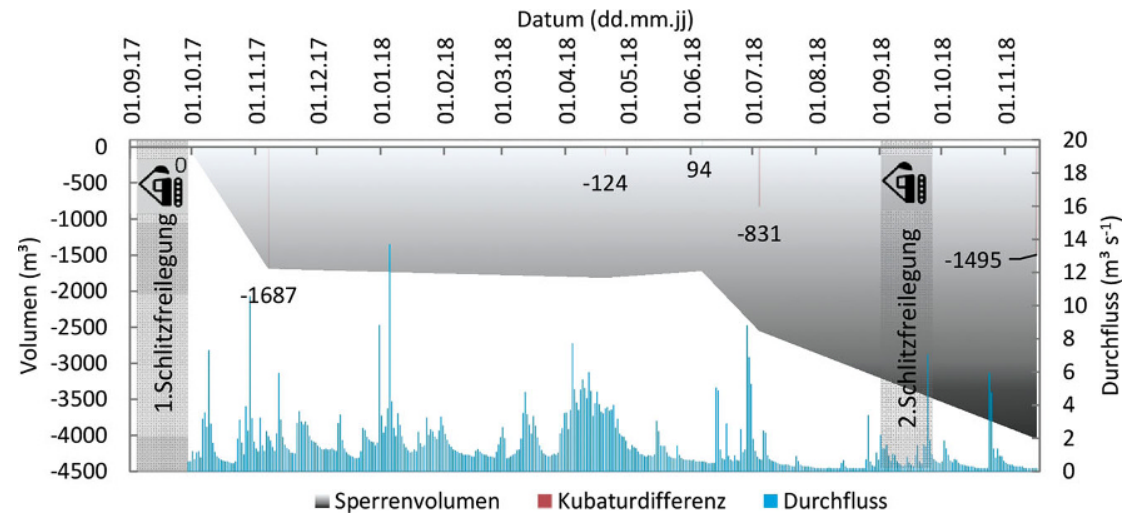

Abb. 11 Zeitliche Änderung des Sperrenvolumens und Tagesmittelwerte des Durchflusses bei Messstelle M3

gen der Osmoseregulation und/oder des Blutchemismus, Änderung von Wachstumsraten),

- (ii) Auswirkungen auf das Verhalten (z.B. Ausweichen, Veränderung des Fressverhaltens aufgrund eingeschränkter Sichtweite) und

- (iii) Auswirkungen auf den Lebensraum (z.B. verringerte Durchströmung des Interstitials und damit einhergehende Unterversorgung von Fischeiern mit Sauerstoff, Abnahme an benthischen Invertebraten und
Als ökologisch optimaler Zeitpunkt für eine Schlitzfreilegung an der Sperre wurden die Monate Juni bis September ausgewählt. Dabei wurde angenommen, dass die Zeit von Beginn der Laichzeit (Oktober) bis zum Ende des Larvenstadiums (Anfang Juni) die sensibelste Lebensphase für die Fortpflanzung der Bachforelle darstellt (Abb. 9). Beide Schlitzfreilegungen wurden deshalb im September vor Beginn der Laichzeit durchgeführt.

Um mögliche Auswirkungen durch die an der Geschiebesperre gesetzten Maßnahmen (Totholzräumungen) auf die im Strobler Weißenbach vorkommende Fischpopulation dokumentieren bzw. bewerten zu können, wurden neben dem Monitoring der Schwebstoffkonzentration auch Watbefischungen gemäß der Gewässerzustandsüberwachungsverordnung (GZÜV, BGBI. II Nr. 479/2006 i.d. g. F.) durchgeführt. Dabei wurde zuerst in einem fischökologischen Premonitoring der IstZustand der Fischfauna am 12.09.2017 mittels Elektrobefischung als Referenzzustand erhoben. Am 18.09.2018 fand eine zweite Befischung im Rahmen des Postmonitorings statt. Insgesamt wurde der Fischbestand in drei verschiedenen Teilstrecken des Strobler Weißenbaches erhoben (siehe Abb. 1).

\section{Ergebnisse}

\subsection{Sperrenvolumen}

Durch die Befliegungen der Sperre und die fotogrammetrische Auswertung der Luftbilder konnten hochauflösende Orthofotos, Geländemodelle (3D) und Differenzenkarten erstellt werden (Abb. 10).

Durch Auswertung der Differenzenplots erhält man Informationen über die $\mathrm{Zu}$ - sowie Abnahme des Sperrenvolumens (siehe Abb. 11). Dadurch konnte festgestellt werden, dass nach der ersten Totholzräumung (29.09.2017) bis zur 2. Befliegung (07.11.2017) die Kubatur innerhalb der Sperre um ca. $1700 \mathrm{~m}^{3}$ abnahm (Abb. 11). Im Zeitraum vom 07.11.2017 bis 06.06.2018 stellte sich nahezu ein Gleichgewicht innerhalb der Sperre ein. Zwischen 06.06.2017 und 04.07.2018 kam es zum zweitgrößten Abflussereignis im Jahr 2018 (Tagesmittel von $8,8 \mathrm{~m}^{3} \mathrm{~s}^{-1}$ am 28.06.2018 bzw. $Q_{\max }=18,7 \mathrm{~m}^{3} \mathrm{~s}^{-1}$ ), aus dem eine Selbstentleerung von ca. $800 \mathrm{~m}^{3}$ resultierte. Die zweite Totholzräumung erfolgte am 11.09.2018. Hier wurde der 


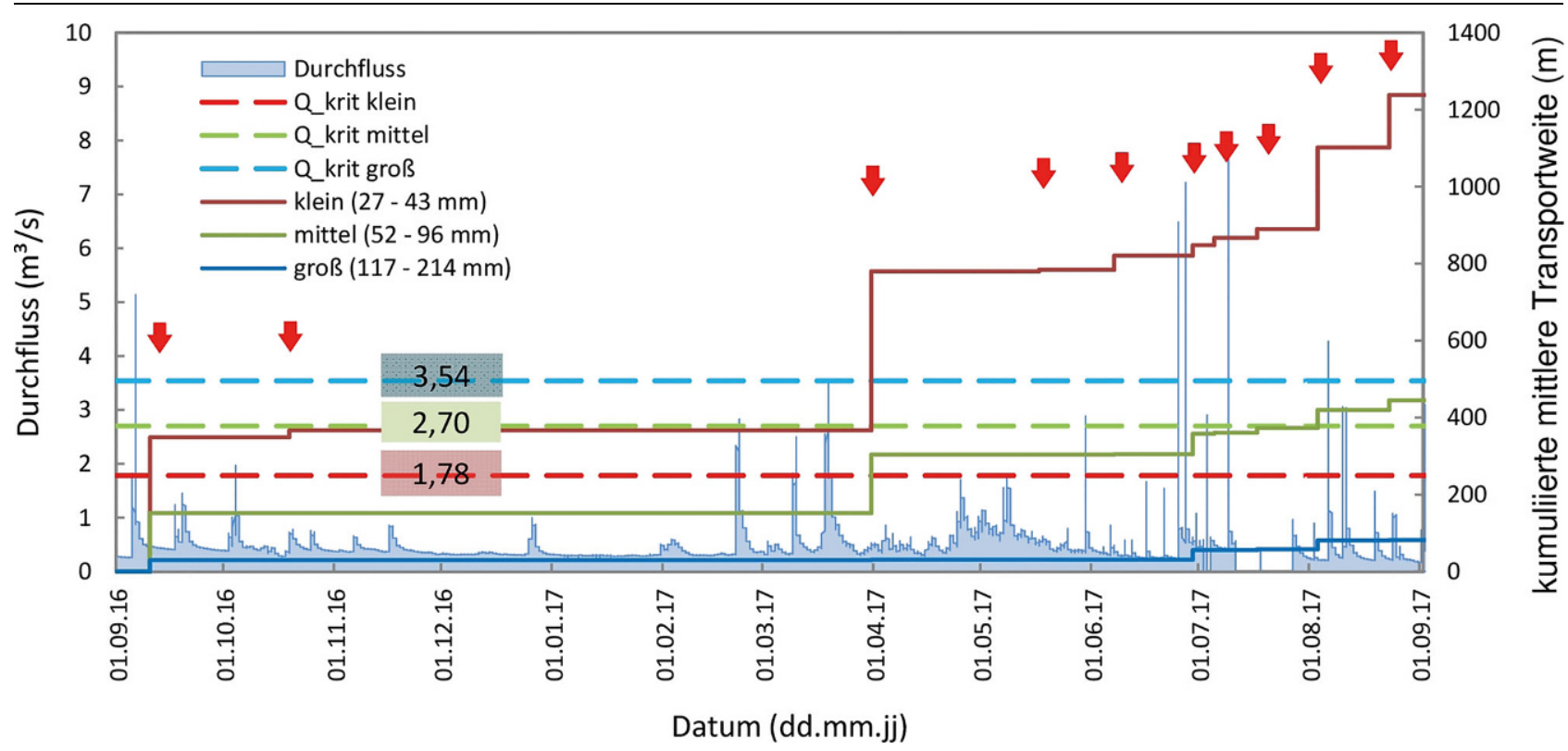

Abb. 12 Transportweiten-Durchfluss-Beziehung

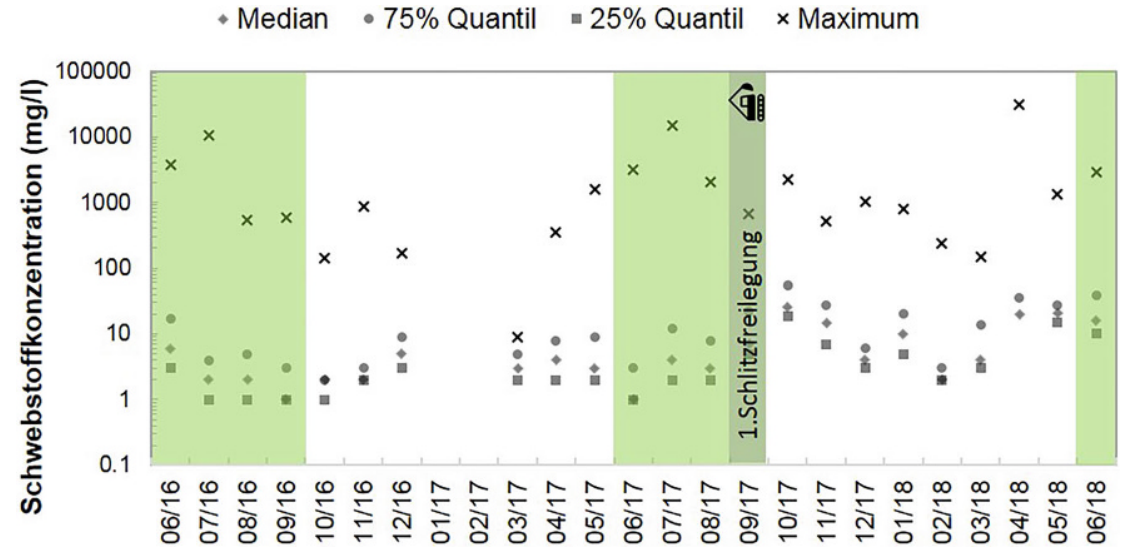

Abb. 13 Median, 75-\%-Quantil, 25-\%-Quantil und Maximum der Schwebstoffkonzentration pro Monat an der Messstelle 2 basierend auf einem Aufzeichnungsintervall von $15 \mathrm{~min}$

Sperrenschlitz erneut bis zu einer Tiefe von ca. 1,5 Metern freigelegt. Im Zuge dieser Maßnahme wurden zusätzlich auch ca. $100 \mathrm{~m}^{3}$ Material aus der Sperre entnommen und ca. $2 \mathrm{~km}$ unterhalb der Sperre im Uferbereich des Strobler Weißenbachs wieder eingebracht. Im Zeitraum der 2. Schlitzfreilegung vom 04.07.2018 bis zum 16.11.2018 kam es somit zu einem Kubatur-Rückgang von ca. $1500 \mathrm{~m}^{3}$. Hier ist anzumerken, dass zeitlich unmittelbar vor der Entfernung des Totholzes keine Befliegung durchgeführt wurde. Dadurch kann angenommen werden, dass die verstärkte Verringerung des Volumens nicht mit 04.07.2018, sondern mit 11.09.2018 einsetzte. Im gesamten Untersuchungs- zeitraum Zeitraum vom 29.09.2017 bis 16.11.2018 kam es somit zu einer Volumsreduktion von ca. $4000 \mathrm{~m}^{3}$, eine grobe Schätzung des noch gefüllten Stauraums ergibt ein Volumen von $35.000 \mathrm{~m}^{3}$. Ohne neuerliche Geschiebeeinträge könnte sich bei freiem Schlitz und ähnlicher hydrologischer-Jahreswassermengen der Stauraum somit in ca. neun Jahren selbstständig entleeren.

\subsection{Tracer}

Im Zeitraum vom 31.08 .2016 bis 31.08.2017 fanden zehn DetektionsKampagnen statt, bei denen die Tracersteine mittels mobiler Antenne aufgesucht und deren Positionen vermessen

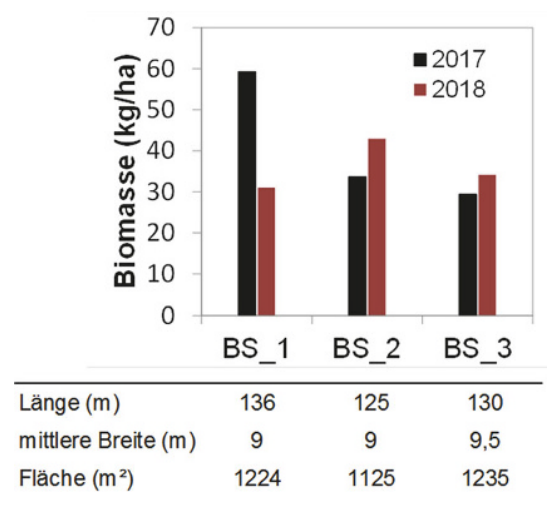

Abb. 14 Nach der Moran-ZippinMethode (Seber and Le Cren 1967) geschätzte Biomasse der Bachforelle (Salmo trutta fario) in den Jahren 2017 und 2018

wurden. Die Untersuchungsstrecke des Tracermonitorings beträgt $3,2 \mathrm{~km}$ und im Schnitt wurden zwei Arbeitstage für die Suche benötigt. Die mittlere Wiederauffindungsrate in dieser Zeitperiode betrug $94 \%$, wobei die geringste Wiederauffindungsrate bei $86 \%$ und die höchste bei $98 \%$ lag. Der Mittelwert der Transportweiten der Klasse „klein“ betrug $1519 \mathrm{~m}$, jener der Klasse „mittel“ $471 \mathrm{~m}$ und jener der Klasse "groß“ $87 \mathrm{~m}$ über den gesamten Untersuchungszeitraum. Abb. 12 zeigt den Zusammenhang zwischen den gemessenen Durchflüssen an Messstelle M4 und den kumulierten Transportweiten dieser drei Klassen. Zusätzlich ist dieser Abbildung der kritische Durchfluss zu 


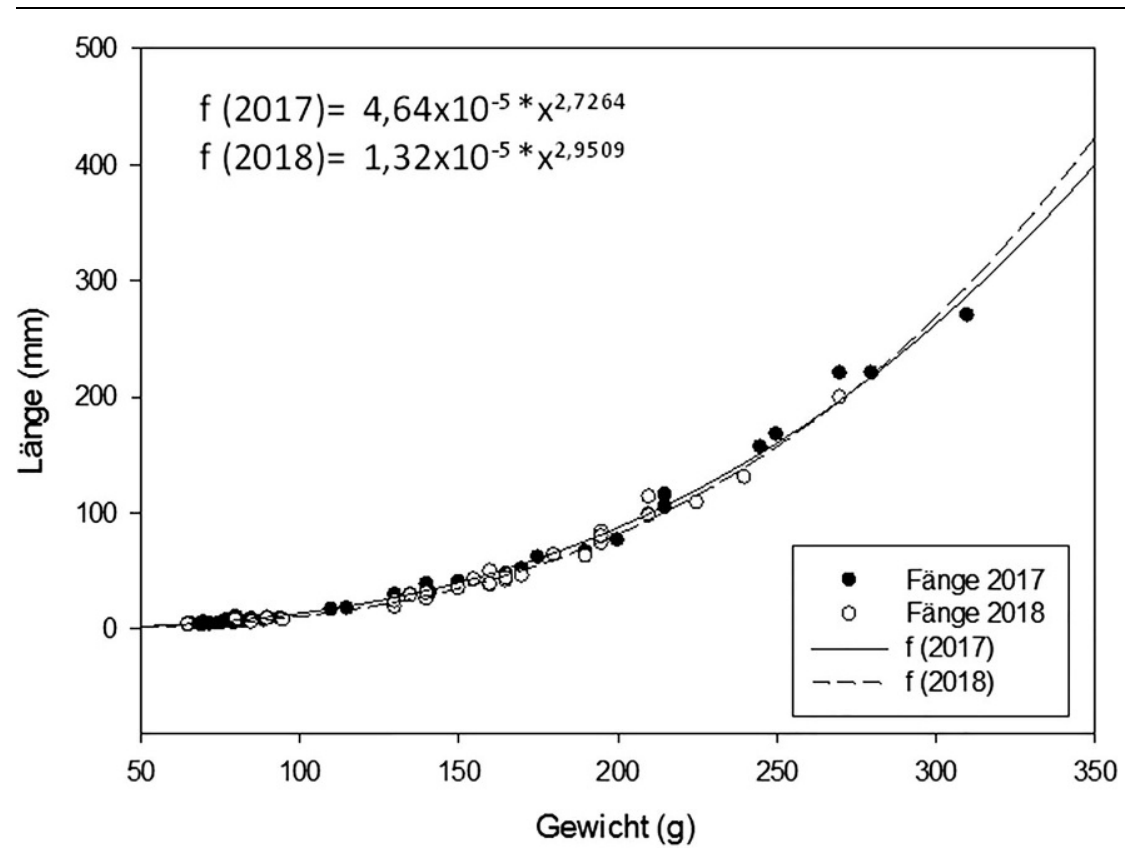

Abb. 15 Vergleich der Längen-Gewichts-Beziehung der in Befischungsstrecke 1 gefangenen Bachforellen (Salmo trutta fario) zwischen 2017 und 2018

entnehmen, ab dem die Tracersteine der jeweiligen Klassen in Bewegung gesetzt werden. Der kritische Durchfluss Q $Q_{\text {krit }}$ wurde über die gemessenen Transportweiten der Tracerdetektionen in Abhängigkeit von den zuvor aufgetretenen Abflussspitzen ermittelt. Jene gemessene Durchflussspitze, bei denen sich einzelne Steine der Fraktionen $\mathrm{zu}$ bewegen begannen, wurde als kritischer Durchfluss definiert. Der kritische Durchfluss der Klasse „klein“ ergab sich dabei bei $1,78 \mathrm{~m}^{3} / \mathrm{s}$, für die Klasse „mittel“ ergab sich ein Qkrit von $2,88 \mathrm{~m}^{3} / \mathrm{s}$ und für die Klasse "groß“ ein $Q_{k r i t}$ von $3,54 \mathrm{~m}^{3} / \mathrm{s}$. Wird der kritische Durchfluss überschritten, kommt es zum Zeitpunkt der Tracerdetektion (roter Pfeil) zu einem Sprung in der Linie der kumulierten Transportweite, was auf einen Transport der Tracersteine schließen lässt. Zwischen 8 . und 9. Tracerdetektion kam es $\mathrm{zu}$ einem Ausfall des Pegels an Messtelle M4, daher sind für diesen Zeitraum keine Aufzeichnungen vorhanden (Lücke in der Durchflussganglinie im Juli 2017). Im Vergleich zu späteren Detektionen wurden nach dem ersten relevanten Abflussereignis nach der Ausbringung der Tracer in allen Tracerklassen relativ hohe Transportweiten festgestellt. Im Gegensatz dazu nehmen die Transportweiten mit steigender Anzahl an Transportereignissen in Relation zum gemessenen Abfluss ab.
Aufgrund der erhobenen Tracerdaten und der mittels eines HEC-RASModells ermittelten mittleren Sohlschubspannungen konnte mithilfe des HyMoCARES-tools zur Abschätzung der Transportgeschwindigkeit von zugegebenem oder remobilisiertem Geschiebe die generalisierte Formel zur Berechnung der virtuellen Geschwindigkeit für den Strobler Weißenbach ermittelt werden. Bestimmt werden hierbei aufgrundlage der gemessenen Transportweiten und der ermittelten Sohlschubspannungen die Koeffizienten a und der Shields-Parameter $\tau^{*}$ crit, welche in die Formel eingefügt werden. Der Koeffizient a wird durch Division mit der Sinkgeschwindigkeit der Partikel zum dimensionslosen Faktor a* (Klösch and Habersack 2018).

Gl. 1: Formel zur Transportgeschwindigkeit von Klösch and Habersack 2018, kalibriert mit Daten des Strobler Weißenbachs:

$$
\begin{gathered}
V_{u}=0,504\left(\frac{d_{i}}{d_{50}}\right)^{1,182} \sqrt{g d_{i} \frac{\rho_{s}-\rho}{\rho}} \\
{\left[\frac{\tau}{\left(\rho_{s}-\rho\right) g d_{i}}-0,081\left(\frac{d_{i}}{d_{50}}\right)^{-0,737}\right]^{\frac{3}{2}}}
\end{gathered}
$$

$\mathrm{d}_{50}=$ Mediandurchmesser

$d_{i}=$ charakteristischer Korndurchmesser

$\mathrm{g}=$ Erdbeschleunigung $\rho_{\mathrm{s}}=$ Dichte des Steins

$\rho=$ Dichte von Wasser

$\tau=$ Sohlschubspannung

\subsection{Schwebstoffmonitoring}

Die gemessene Schwebstoffkonzentration an der Messstelle 2 ist in Abb. 13 als Maximum, 75-\%-Quantil, Median und 25-\%-Quantil pro Monat für den Zeitraum von zwei Jahren dargestellt. Datengrundlage für diese Auswertung sind Aufzeichnungen der Trübungssonde Solitax ts-line (Fa. Hach Lange) im 15-Minuten-Intervall. Der Zeitraum von Juni 2016 bis September 2017 zeigt die natürliche Bandbreite der auftretenden Schwebstoffkonzentrationen vor der ersten Schlitzfreilegung (Totholzräumung). Auffallend - in der Zeit des Premonitorings - sind zwei Extremereignisse im Juli 2016 und im Juli 2017 mit einer Schwebstoffkonzentration von ca. $10.600 \mathrm{mg} / \mathrm{l}$ bzw. $14.500 \mathrm{mg} / \mathrm{l}$. Betrachtet man jedoch das 75-\%-Quantil der Monatswerte, so zeigt sich, dass in den selben Monaten $75 \%$ aller gemessenen Werte eine Konzentration von $4 \mathrm{mg} / \mathrm{l}$ bzw. $12 \mathrm{mg} / \mathrm{l}$ nicht überschritten haben. Im Postmonitoring erhöht sich das gemessene Monatsmaximum (April 2018) auf ca. $30.400 \mathrm{mg} / \mathrm{l}$. Die höchsten Werte hinsichtlich des 75\%-Quantils traten im Folgemonat der Schlitzfreilegung (Oktober 2017) mit $55 \mathrm{mg} / \mathrm{l}$ und im Juni $2018 \mathrm{mit} 38 \mathrm{mg} / \mathrm{l}$ auf.

\subsection{Biotik}

In Abb. 14 sind die Längen-GewichtsBeziehungen der in BS_1 gefangenen Bachforellen aus Pre- und Postmonitoring dargestellt. Ein Vergleich beider Regressionskurven zeigt keinen nennenswerten Unterschied (Abb. 15). Eine permanente Verschlechterung des physiologischen Zustands (Kondition) der gefangenen Fische aufgrund von verringerter Nahrungsverfügbarkeit bzw. aufgrund einer langfristigen Reduktion der Nahrungsaufnahme oder des Fresserfolgs (reduzierte Sichtweite) kann daher ausgeschlossen werden. Bei einer Reduktion der Wachstumsraten würde die Regressionskurve (Potenzfunktion) aus Länge und Gewicht aus dem Jahr 2018 merkbar unter jener von 2017 liegen.

Der Vergleich der Befischungsergebnisse in BS_1 zeigt einen Rückgang der Bachforellenbiomasse von $59,3 \mathrm{~kg} / \mathrm{ha}$ 

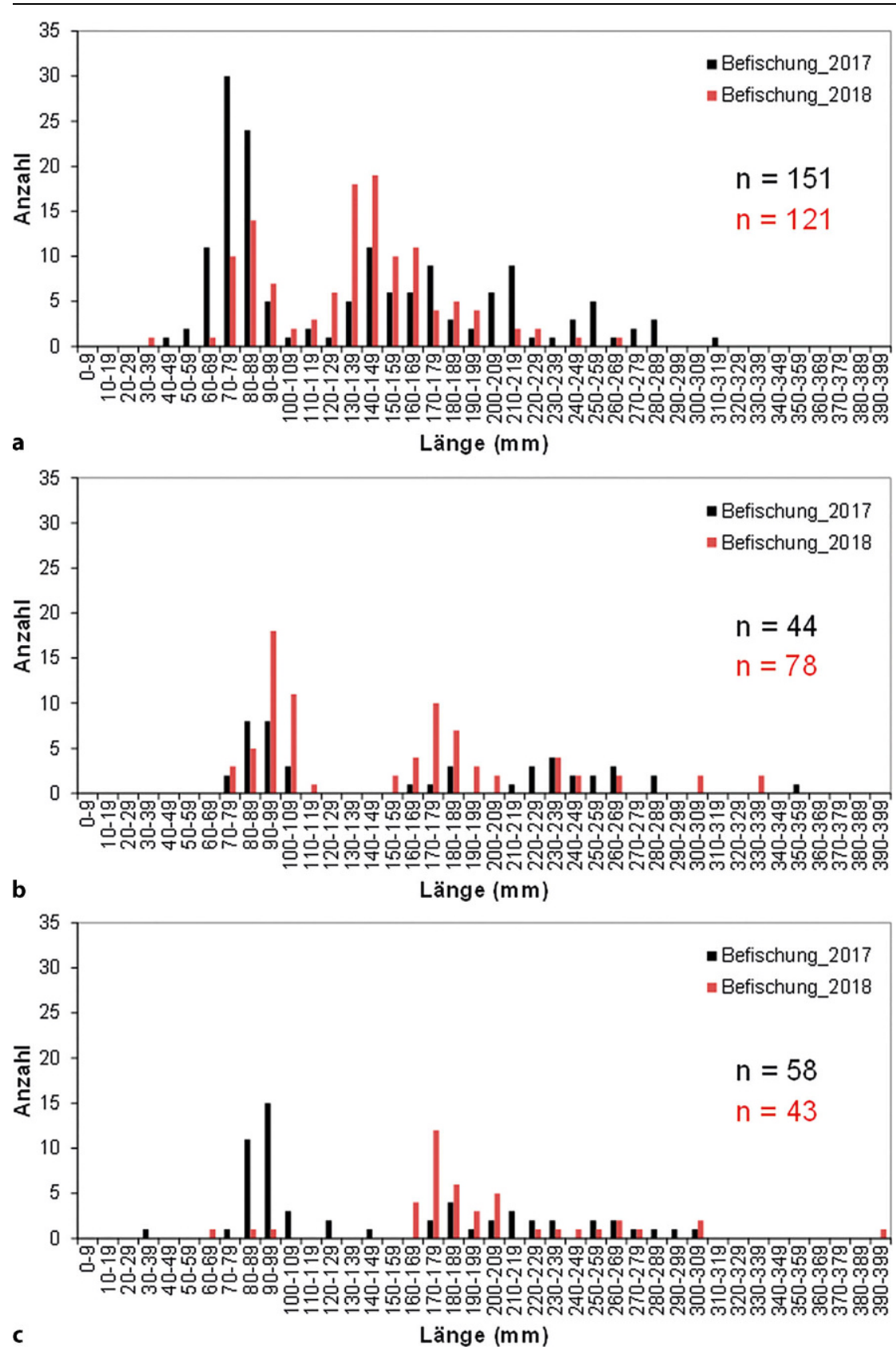

Abb. 16 Längenfrequenzdiagramm der Bachforelle von (a) Befischungsstrecke 1 (BS_1), (b) Befischungsstrecke 2 (BS_2) und (c) Befischungsstrecke 3 (BS_3) in den Jahren 2017 und 2018

auf $31,1 \mathrm{~kg} / \mathrm{ha}$. Hingegen konnte in BS_2 sowie in BS_3 eine Zunahme hinsichtlich der Biomasse im Vergleich zum Vorjahr verzeichnet werden. Diese stieg in BS_2 von 33,7 auf $42,9 \mathrm{~kg} / \mathrm{ha}$ und in BS_3 von 29,4 auf $34,2 \mathrm{~kg} / \mathrm{ha}$ an.

Betrachtet man die Längenfrequenzdiagramme der Bachforelle aus dem Jahr 2017 und 2018 für die BS_1, so zeigt sich eine Abnahme innerhalb der Gruppe von 0+ und 2+ Fischen. Die Anzahl der 1+ Fische liegt im Jahr 2018 genen 0+ Bachforellen. Im Bereich der 1+ Fische konnte hingegen ein Anstieg verzeichnet werden. Die Anzahl an gefangenen Individuen sank in BS_3 von 58 auf 43 (Abb. 16).

In den beiden unten liegenden Befischungsstrecken 2 und 3 konnten sowohl im Jahr 2017 als auch im Jahr 2018 Koppen (Cottus gobio) gefangen werden. Obwohl den Kieslückenraum bewohnende Arten wie die Koppe durch die Elektrobefischung quantitativ schwer zu erfassen sind, zeigen die Längenfrequenzdiagramme beider Strecken ein ähnliches Bild im Jahresvergleich (Abb. 17). Außerdem kam es in beiden Strecken zu einer marginalen Zunahme bei der Anzahl gefangener Individuen $(+2$ bzw. +5$)$.

Für die Interpretation der Daten aus BS_1 ist der Umstand maßgeblich, dass aufgrund eines (für Fische) unüberwindbaren Querbauwerks (Sohlstufe) unterhalb dieser Befischungsstelle (ca. $3 \mathrm{~km}$ ) keine Einwanderung aus dem Unterlauf des Strobler Weißenbaches bzw. aus der Ischl erfolgen kann. Alle in BS_1 gefangenen Individuen waren somit sämtlichen Extremereignissen hinsichtlich der Schwebstoffkonzentration ausgesetzt. Zusätzlich muss an dieser Stelle erwähnt werden, dass fischereiwirtschaftliche Besatzmaßnahmen im vorliegenden Betrachtungszeitraum ausschließlich im Jahr 2017 in Form von $0+$ Bachforellen stattgefunden haben. Im Gegensatz zu 2017 stammen daher die in 2018 gefangenen 0+ Bachforellen ausschließlich aus natürlicher Eigenreproduktion. Dies ist bei der Interpretation der Befischungsergebnisse aller Strecken zu berücksichtigen.

Weiters ist $\mathrm{zu}$ berücksichtigen, dass für die Biomasse vor allem die größeren Adulttiere entscheidend sind, da diese gewichtsmäßig naturgemäß den größten Anteil an der Gesamtbiomasse aufweisen. Das heißt, die An- oder Abwesenheit einzelner Individuen dieser Größe kann die errechnete Biomasse vor allem in kurzen Untersuchungsstrecken (Abb. 18) maßgeblich beeinflussen. Die Abnahme an Adultfischen in BS_1 könnte neben der Entnahme aus der Sportfischerei auch auf ein Zusammenspiel aus Anlandungen innerhalb der Kolke (Adulthabitat) bei gleichzeitiger Niederwasserführung am Befischungstag $\left(Q_{\text {pre }}=0,8 \mathrm{~m}^{3} \mathrm{~s}^{-1} ; \quad Q_{\text {post }}=0,3 \mathrm{~m}^{3} \mathrm{~s}^{-1}\right) \quad \mathrm{zu}-$ rückzuführen sein. Eine Abnahme der Kolktiefe konnte innerhalb von BS_1 im Rahmen des Projekts bereits vor der Be- 

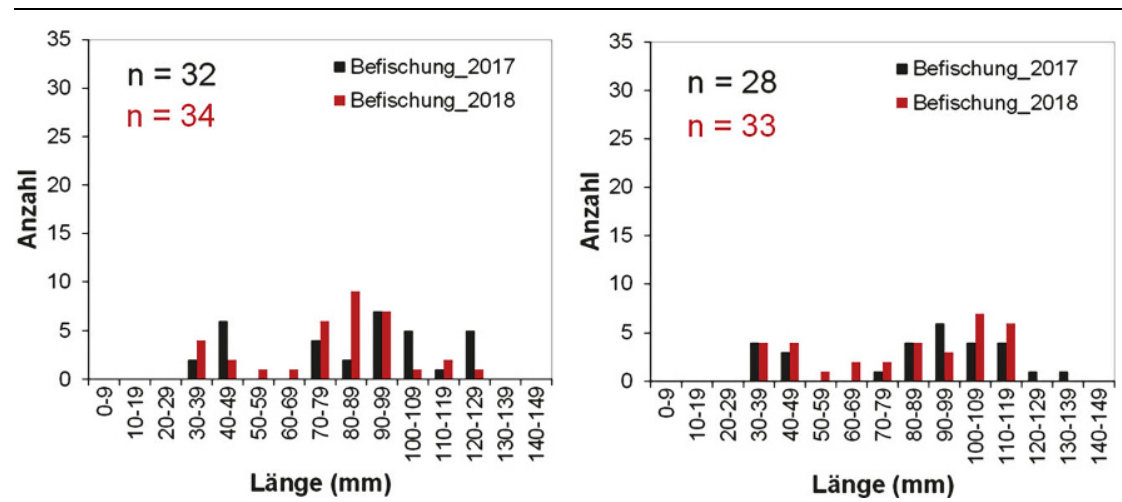

Abb. 17 Längenfrequenzdiagramm der Koppe von Befischungsstrecke 2 (links) und Befischungsstrecke 3 (rechts) in den Jahren 2017 und 2018
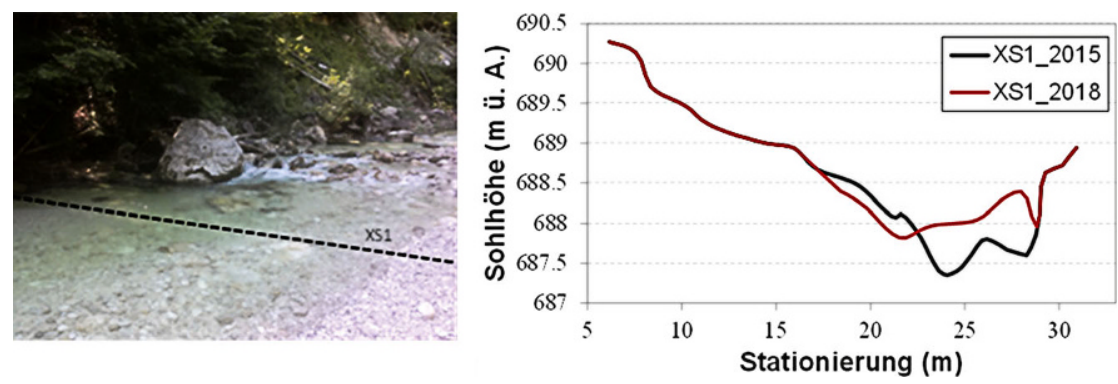

Abb. 18 Links: Lage des Querprofils durch die hydromorphologische Einheit Kolk in BS_1, rechts: Vergleich der Profilvermessung aus 2015 und 2018

fischung dokumentiert und beobachtet werden (vgl. Kolktiefe BS_1 2015/2018 in Abb. 18).

Wäre der Rückgang an größeren Bachforellen auf die Zunahme von Häufigkeit, Dauer oder Intensität von Sedimenttransport zurückzuführen, so würden mit höchster Wahrscheinlichkeit auch die in BS_1 gefangenen 1+ Fische davon betroffen sein bzw. müsste dieser Effekt auch in BS_2 und BS_3 in irgendeiner Form anhand vorliegender Befischungsdaten sichtbar sein. Die nahezu unveränderten Längenfrequenzdiagramme der Koppe in BS_2 und BS_3 unterstützen diese These. Koppen benötigen außerdem relativ groben und durchlässigen Kies für die Reproduktion, daher ist diese Art als sensibel gegenüber Sedimentablagerungen anzusehen (Mills und Mann 1983).

Durch die partielle Freilegung des Schlitzes von Schwemmholz an der Sperre (Totholzräumung) wird gewährleistest, dass der Austrag an Sedimenten aus dem Bauwerk ausschließlich ereignisbezogen, also bei erhöhtem Abfluss, stattfindet. Dies ist insofern von hoher Relevanz, da sich das Ausmaß der schädlichen Wirkung erhöhter Schwebstoffkonzentrationen auf Fische, neben der Schwebstoffkonzentration, zusätz- lich aus der Expositionsdauer zusammensetzt (Newcombe and Jensen 1996). Durch die geringe Größe des Einzugsgebietes (EZG bis zur Sperre $=7,7 \mathrm{~km}^{2}$ ) flussauf des Sperrenbauwerks ist im Rezu rechnen. Weiters kann durch diese Art von Maßnahme über die „Freilegungstiefe“, die Mächtigkeit des für den Austrag freigegebenen Sedimentlayers begrenzt bzw. bestimmt werden.

\section{Schlussfolgerung}

Am Strobler Weißenbach, einem typischen Kalkschuttwildbach und Zubringer zur Ischler Ache wurden nach Hochwasser- und Geschiebetransportereignissen in den 1970er-Jahren geschiebebindende Schutzbauwerke in Form von Schlitzsperren errichtet. Die Schlitze hatten das Ziel, die Feststoffe wie Geschiebe und Wildholz im Ereignisfall zurückzuhalten und bei kleineren Ereignissen wieder an den Unterlauf abzugeben. Verklausungen der Schlitze mit Wildholz/Totholz führten zu einem fast gänzlichen Rückhalt, wodurch sich die Verlandungsräume der Sperren immer mehr füllten. Neben der maschinellen und kostenintensiven Räumung zur Wiederherstellung der Schutzfunkgelfall mit Ereignissen von kurzer Dauer tion sind aufgrund der fehlenden Sedimentdurchgängigkeit auch ökologische und sedimentologische Veränderungen im Unterlauf und bis in den Vorfluter die Folge. Zur Optimierung der Gesamtsituation sind Kenntnisse über die Wildbachprozesse im Jahresgang sowie bei Extremereignissen notwendig, sodass einerseits die schutztechnischen Anforderungen erfüllt und andererseits auch die ökologischen sowie sedimentologischen Anforderungen im Unterlauf sowie Vorfluter berücksichtigt werden.

Die Messungen innerhalb der dreijährigen Monitoringphase lieferten wichtige Daten über Abfluss, Schwebstoffkonzentrationen, die Transportweiten von Tracersteinen, die Selbstentleerungsraten nach Totholzentfernung am Schlitz an der Kammersbachsperre und die Änderungen der Habitate sowie der Biomasse im Monitoringzeitraum. Folgende Ergebnisse und Schlussfolgerungen sind ableitbar:

1. Volumen der Sedimentzugabe aufgrund der manuellen Öffnung des Schlitzes der Kammersbachsperre (ausgeräumtes Material je Maßnahme).

2. Sediment-/Geschiebetransportweiten der verschiedenen Kornfraktionen (Transportweiteformel für den Strobler Weißenbach).

3. Bandbreite der aufgetretenen Schwebstoffkonzentrationen sowohl im Pre- als auch im Postmonitoring.

4. Abschätzung eines ökologisch optimalen Zeitpunkts für eine Schlitzfreilegung an der Sperre.

5. Änderung der Biomasse der Bachforelle im Monitoringzeitraum.

Ad. 1 Das maximale Sedimentvolumen wurde nach der ersten Totholzräumung bei der Kammersbachsperre remobilisiert und betrug ca. $1700 \mathrm{~m}^{3}$. Bei der zweiten Schlitzfreilegung kam es zu einer Kubaturreduktion von ca. $1500 \mathrm{~m}^{3}$. Dadurch kann angenommen werden, dass bei ähnlichen hydrologischen Verhältnissen pro Schlitzfreilegung das Sperrenvolumen um ca. $1600 \mathrm{~m}^{3}$ abnimmt.

Ad. 2 Unkontrollierte Zugaben auf der flussabliegenden Seite von Sperren führen häufig zu temporären Anlandungen. Das vorwiegend feinkörnige Sediment wird bereits bei leicht erhöhten Abflussverhältnissen mobilisiert und bildet eine Art Geschiebeband, welches sukzessive flussabwärts „wandert“ (Liedermann et al. 2013). Durch die für den Strobler Weißenbach kali- 
brierte Transportweitenformel kann für jede Kornfraktion die mittlere Transportweite in Abhängigkeit vom Durchfluss berechnet werden bzw. die Frage geklärt werden, ab wann das zugegebene Material den Vorfluter (Ischler Ache) in Form von Geschiebe erreicht.

Die teilweise ausgeprägte Deckschicht der Bachsohle wird dabei vorübergehend vom freigesetzten Geschiebe überlagert, was zu einer Verringerung der Abflusskapazität (infolge der höheren Sohllagen) und einer Dynamik der Fischhabitate (sowohl positiv als auch negativ) führen kann. Die Intensität dieser Geschiebeumlagerung und somit das Ausmaß der Überlagerung ist dabei von Art und Ausmaß der Geschiebefreisetzung abhängig.

Derartige Geschiebeumlagerungen ergeben sich allerdings nicht nur durch menschliche Eingriffe, sondern spiegeln durchaus die natürliche Fließgewässerdynamik wider. Ähnlich einer maschinellen Verkippung führen auch natürliche Geschiebeeinträge (beispielsweise Murgänge) $\mathrm{zu}$ einer plötzlichen Bereitstellung von großen Mengen an Sediment. Um nun die Auswirkungen der menschlich verursachten Geschiebe- und Schwebstoffbelastung beurteilen zu können, muss diese in die Spannweite der natürlichen Dynamik eingeordnet werden.

Ad. 3 Die höchsten Schwebstoffkonzentrationen mit etwa $30.000 \mathrm{mg} / \mathrm{l}$ traten im Postmonitoring infolge eines natürlichen Ereignisses auf, lagen jedoch über den Konzentrationen des Premonitorings, was als Folge der höheren Verfügbarkeit an Sedimenten zurückzuführen sein könnte. Hinsichtlich der ökologischen Auswirkung solcher Konzentrationen ist hier zu berücksichtigen, dass diese in derartigem Ausmaß am Strobler Weißenbach zeitlich sehr kurz auftreten $(<15 \mathrm{~min})$.

Ad. 4 Als ökologisch optimaler Zeitpunkt für eine Schlitzfreilegung an der Sperre wurden die Monate Juni bis September ausgewählt. Dabei wurde angenommen, dass die Zeit von Beginn der Laichzeit (Oktober) bis zum Ende des Larvenstadiums (Anfang Juni) die sensibelste Lebensphase für die Fortpflanzung der Bachforelle darstellt (Abb. 9). Beide Schlitzfreilegungen wurden deshalb im September vor Beginn der Laichzeit durchgeführt. Durch natürliche Schwebstoffkonzentrationen und eine durchflussabhängige SedimentRemobilisierung können unnatürlich hohe Belastungen vermieden und Aus- wirkungen auf die Fischerei und andere Wassernutzer auf ein natürliches Maß reduziert werden.

Ad. 5 Ein fischökologisches Monitoring vor und nach Freilegung des Sperrenschlitzes zeigte keine signifikanten Änderungen in der Biomasse der Bachforelle an den beiden im Unterlauf gelegenen Befischungsstrecken. In der oben gelegenen Untersuchungsstrecke kam es jedoch zu einer Halbierung der Biomasse aufgrund der Abwesenheit einiger weniger Adulttiere. Dieser Rückgang wird aber nicht auf die Änderung der aufgetretenen Schwebstoffkonzentrationen, sondern vielmehr auf die morphologische Veränderung (Habitatverfügbarkeit) innerhalb der Befischungsstelle 1 (Teilverfüllung eines Kolks) aufgrund einer erhöhten Sedimentverfügbarkeit zurückgeführt. Weiters konnte ein Rückgang der 0+ Bachforellen in Befischungsstrecke 1 und 3 festgestellt werden, was möglicherweise auch mit einem Ausbleiben von Besatz mit 0+ Fischen im Jahr 2018 in Zusammenhang steht.

\section{Ausblick}

Die Daten aus dem bisherigen Monitoring und die daraus abgeleiteten Erkenntnisse liefern wichtige Grundlagen für die Erstellung eines Geschiebebewirtschaftungskonzepts am Strobler Weißenbach. Eine Weiterführung der bisherigen Messungen ist zur Absicherung der aufgestellten Thesen sinnvoll und notwendig. Es geht dabei aber nicht nur um geschieberelevante Fragestellungen und ökologische Verträglichkeit, sondern auch um gesellschaftliche Akzeptanz. Die Beeinträchtigung etwaiger Wassernutzer (beispielsweise Fischereiwirtschaft) sollte also möglichst gering gehalten werden.

Zur Gewährleistung dieser Anforderungen ist ein geeignetes Konzept zur Sedimentbewirtschaftung (Sperrenmanagement) notwendig, welches Zeitpunkt sowie Art und Intensität der Räumungsmaßnahmen regelt. Erfahrungswerte dazu sind allerdings nur spärlich vorhanden und für die Erarbeitung eines solchen Konzepts fehlt es bisher an fundierten Untersuchungen. Um negative Auswirkungen auf Ökologie und lokale Wassernutzer zu verhindern, wird ein Konzept erarbeitet, das eine naturnahe Sedimentdotation ermöglicht und das Ausmaß der Wassertrübung und Geschiebetransportraten innerhalb der natürlichen Bandbreite für die Organis- men hält. Dies setzt voraus, dass diese Maßnahmen nur während erhöhter Wasserführungen wirken.

Die Geschiebebewirtschaftung des Strobler Weißenbaches soll als Pilotprojekt für eine kontrollierte Wiedereinbringung von Räumgut (Sedimente und Geschiebe) dienen. So können die Erkenntnisse, die aus diesem Projekt gezogen werden, Grundlagen für die Geschiebebewirtschaftung (Zugabe von Sedimenten) in ähnlichen alpinen Einzugsgebieten werden. Ziel ist es, nach Abschluss des Projekts einen Leitfaden für ein generelles Räumungskonzept zu erstellen.

Hierfür werden in den nächsten drei Jahren weitere Versuche zur Wiedereinbringung von Sedimenten am Strobler Weißenbach durchgeführt. Außerdem wird es zukünftig von Bedeutung sein, die Feststoffdynamik über den unmittelbaren Einflussbereich im Wildbach hinaus zu betrachten bzw. zu bewerten. Für das hier präsentierte Beispiel des Strobler Weißenbachs bedeutet dies, dass die an die natürliche Situation angepasste Geschiebebewirtschaftung mitunter kurzfristig/temporär zu einer Verschlechterung bestimmter Habitatverhältnisse führen kann (z.B. Verfüllung der Kolke). Die eigendynamische Erneuerung von Kieslaichplätzen durch die verbesserte Geschiebedynamik wirkt aber weit über das Einzugsgebiet des Wildbachs hinaus. An dem gewählten Fallbeispiel ist der Strobler Weißenbach als wesentlicher Lieferant für Kieslaichplätze für die Ischl, die aus einem See entspringt, zu nennen. Weiters ist die Bedeutung der Feststoffdynamik für die Laichplätze und damit auch für die nachhaltige fischereiliche Bewirtschaftung der Traun (aus dem Hallstätter See kommend) zu nennen.

Funding Open access funding provided by University of Natural Resources and Life Sciences Vienna (BOKU).

Open Access Dieser Artikel wird unter der Creative Commons Namensnennung 4.0 International Lizenz (http:// creativecommons.org/licenses/by/4. $0 /$ deed.de) veröffentlicht, welche die Nutzung, Vervielfältigung, Bearbeitung, Verbreitung und Wiedergabe in jeglichem Medium und Format erlaubt, sofern Sie den/die ursprünglichen $\mathrm{Au}$ tor(en) und die Quelle ordnungsgemäß nennen, einen Link zur Creative Commons Lizenz beifügen und angeben, $\mathrm{ob}$ Änderungen vorgenommen wurden. 
Originalarbeit

Literatur

Bash, J., Berman, C. H. and Bolton, S. (2001): Effects of turbidity and suspended solids on salmonids, University of Washington Water Center. BMLFUW (2017): Nationaler Gewässerbewirtschaftungsplan 2015 - NGP 2015.URL: https:// www.bmnt.gv.at/wasser/wisa/fachinformation/ ngp/ngp-2015/karten/OW/ow-typologie.html (abgerufen am 17.12.2018).

Nathan Bradley, D. and Tucker, G. E. (2012): Measuring gravel transport and dispersion in a mountain river using passive radio tracers. Earth Surface Processes and Landforms 37(10), 1034-1045.

Chapuis, M., Bright, C. J., Hufnagel, J. and MacVicar, B. (2014): Detection ranges and uncertainty of passive Radio Frequency Identification (RFID) transponders for sediment tracking in gravel rivers and coastal environments. Earth Surface Processes and Landforms 39(15), 2109-2120.

Habersack, H., Huber-Humer, M., Wagner, B. Mosbauer, P., Hrad, M., Fuhrmann, M., Aigner, J. and Klösch, M. (2018): Qualität von Räummaterial aus Wildbächen: Entwicklung einer Methodik für ein vereinfachtes Prüfverfahren, p. 197, Bundesministerium für Nachhaltigkeit und Tourismus, Abteilung III/5, Wildbach- und Lawinenverbauung, Wien.

Habersack, H., Wagner, B., Schoder, A. and

Hauer, C. (2013): The importance of sediment regime and continuity for a sustainable use of hydroelectric power. Österreichische Wasserund Abfallwirtschaft 65(9), 354-361.

Klösch, M. and Habersack, H. (2018): Deriving formulas for an unsteady virtual velocity of bedload tracers. Earth Surface Processes and Landforms 43(7), 1529-1541.

Liedermann, M., Tritthart, M. and Habersack, H. (2013): Particle path characteristics at the large gravel-bed river Danube: results from a tracer study and numerical modelling. Earth Surface Processes and Landforms 38(5), 512-522.

Mills, C. A., Mann R. H. K. (1983): The bullhead Cottus gobio, a versatile and successful fish. Annual Reports of the freshwater Biological Association $51,76-88$.

Newcombe, C. P. and Jensen, J. O. (1996): Channel suspended sediment and fisheries: a synthesis for quantitative assessment of risk and impact. North American Journal of Fisheries Management 16(4), 693-727.

Papež, J., Moser, M., Jager, G., Pitton, G., Recking,

A., Silvestro, C., Bodrato, G., Tresso, F., Del Ves- co, R., Sodnik, J., Klabus, A., Klemenčič, A. K., Jeriček, Š. L. and Mrak, S. (2015): Report on improved concepts of responses of torrent/ river control structures to floods and debris flow impacts (including wood), p. 191, SEDALP.

Phillips, C. and Jerolmack, D. (2014): Dynamics and mechanics of bed-load tracer particles. Earth Surface Dynamics 2(2), 513.

Rindler, R., Kammerlander, J., Moser, M., Holzapfel, P., Hauer, C. and Habersack, H. (2016): Ökologisch verträgliche Sedimentbewirtschaftung am Strobler Weißenbach. Wildbach-, Lawinen-,Erosions- und Steinschlagschutz 177 (Feststofftransport und Sedimentmanagement Sediment Transport and Management).

Rudolf-Miklau, F. and Patek, M. (2004): Geschiebebewirtschaftung in Wildbacheinzugsgebieten im Einklang mit der EU-Wasserrahmenrichtlinie, Riva / Trient.

Seber, G. and Le Cren, E. (1967): Estimating population parameters from catches large relative to the population. The Journal of Animal Ecology, 631-643.

Suda, J., Skolaut, C., Bergmeister, K., RudolfMiklau, F. and Hübl, J. (2008): Zement\&Beton, p. 10, Wien. 